\title{
APPROXIMATION OF FUNCTIONS \\ BY A NEW CLASS OF LINEAR OPERATORS
}

G. C. JAIN

(Received 24 April 1970)

Communicated by B. Mond

\section{Introduction}

Various extensions and generalizations of Bernstein polynomials have been considered among others by Szasz [13], Meyer-Konig and Zeller [8], Cheney and Sharma [1], Jakimovski and Leviatan [4], Stancu [12], Pethe and Jain [11]. Bernstein polynomials are based on binomial and negative binomial distributions. Szasz and Mirakyan [9] have defined another operator with the help of the Poisson distribution. The operator has approximation properties similar to those of Bernstein operators. Meir and Sharma [7] and Jain and Pethe [3] deal with generalizations of Szasz-Mirakyan operator. As another generalization, we define in this paper a new operator with the help of a Poisson type distribution, consider its convergence properties and give its degree of approximation. The results for the Szasz-Mirakyan operator can easily be obtained from our operator as a particular case.

\section{The operator and its convergence}

The operator and its convergence are based on the following two lemmas:

Lemma 1. For $0<\alpha<\infty$, $|\beta|<1$, let

$$
\omega_{\beta}(k, \alpha)=\alpha(\alpha+k \beta)^{k-1} e^{-(\alpha+k \beta)} / k ! ; k=0,1,2, \cdots
$$

then

$$
\sum_{k=0}^{\infty} \omega_{\beta}(k, \alpha)=1
$$

It may be mentioned that (2.1) is a Poisson-type distribution which has been considered by Consul and Jain [2].

The proof of the lemma may be based upon results given by Jensen [5]. If we start with Lagrange's formula 


$$
\phi(z)=\phi(0)+\sum_{k=1}^{\infty} \frac{1}{k !}\left[\frac{d^{k-1}}{d z^{k-1}}\left((f(z))^{k}\right) \phi^{\prime}(z)\right]_{z=0}\left(\frac{z}{f(z)}\right)^{k}
$$

and proceed by setting

we shall get

$$
\phi(z)=e^{\alpha z} \text { and } f(z)=e^{\beta z}
$$

$$
e^{x z}=\sum_{k=0}^{\infty} \alpha(\alpha+k \beta)^{k-1} u^{k} / k !, u=z e^{-\beta z},
$$

where $z$ and $u$ are sufficiently small such that $|\beta u|<e^{-1}$ and $|\beta z|<1$.

By taking $z=1$, the lemma in (2.2) is obvious.

LemMa 2. Let

$$
S(r, \alpha, \beta)=\sum_{k=0}^{\infty}(\alpha+\beta k)^{k+r-1} e^{-(\alpha+\beta k)} / k !, r=0,1,2, \cdots
$$

and

$$
\alpha S(0, \alpha, \beta)=1 .
$$

Then

$$
S(r, \alpha, \beta)=\sum_{k=0}^{\infty} \beta^{k}(\alpha+k \beta) S(r-1, \alpha+k \beta, \beta),
$$

Proof. It can easily be seen that the functions $S(r, \alpha, \beta)$ satisfy the reduction formula

$$
S(r, \alpha, \beta)=\alpha S(r-1, \alpha, \beta)+\beta S(r, \alpha+\beta, \beta) .
$$

By a repeated use of (2.8), the proof of the lemma is straightforward.

From (2.7) and (2.6) when $\beta<1$ we get

and

$$
S(1, \alpha, \beta)=\sum_{k=0}^{\infty} \beta^{k}=1 /(1-\beta),
$$

$$
S(2, \alpha, \beta)=\sum_{k=0}^{\infty} \frac{\beta^{k}(\alpha+k \beta)}{(1-\beta)}=\frac{\alpha}{(1-\beta)^{2}}+\frac{\beta^{2}}{(1-\beta)^{3}} .
$$

We may now define the operator as

$$
P_{n}^{[\beta]}(f ; x)=\sum_{k=0}^{\infty} \omega_{\beta}(k, n x) f(k / n),
$$

where $1>\beta \geqq 0$ and $w_{\beta}(k, n x)$ has been defined in (2.1).

The parameter $\beta$ may depend on the natural number $n$. It is easy to see that for $\beta=0,(2.11)$ reduces to Szasz--Mirakyan operator [9].

The convergence property of the operator $P_{n}^{[\beta]}(f: x)$ is proved in the following theorem: 
THEOREM (2.1). If $f \in C[0, \infty)$ and $\beta \rightarrow 0$ as $n \rightarrow \infty$ then the sequence $\left\{P_{n}^{[\beta]}(f ; x)\right\}$ converges uniformly to $f(x)$ in $[a, b]$, where $0 \leqq a<b<\infty$.

Proof. Since $P_{n}^{[\beta]}(f ; x)$ is a positive linear operator for $1>\beta \geqq 0$, it is sufficient, by Korovkin's result, to verify the uniform convergence for test functions $f(t)=1, t$ and $t^{2}$.

It is clear from (2.2) that

$$
P_{n}^{[\beta]}(1 ; x)=1 .
$$

Going on to $f(t)=t$ and using (2.9) we have

$$
\begin{aligned}
P_{n}^{[\beta]}(t ; x) & =x n \sum_{k=1}^{\infty} \frac{(n x+\beta k)^{k-1}}{k !} e^{-(n x+\beta k)}\left(\frac{k}{n}\right) \\
& =x S(1, n x+\beta, \beta)=\frac{x}{1-\beta} .
\end{aligned}
$$

Proceeding to the function $f(t)=t^{2}$, it can easily be shown that

$$
\begin{aligned}
P_{n}^{[\beta]}\left(t^{2} ; x\right) & =x n \sum_{k=0}^{\infty} \frac{(n x+\beta k)^{k-1}}{k !} e^{-(n x+\beta k)} \frac{k^{2}}{n^{2}} \\
& =\frac{x}{n}[S(2, n x+2 \beta, \beta)+S(1, n x+\beta, \beta)]
\end{aligned}
$$

and a use of (2.9) and (2.10) yields

$$
P_{n}^{[\beta]}\left(t^{2} ; x\right)=\frac{x^{2}}{(1-\beta)^{2}}+\frac{x}{n(1-\beta)^{3}} .
$$

Thus combining the results of (2.12), (2.13) and (2.14) we have

$$
\operatorname{Lim}_{n \rightarrow \infty} P_{n}^{[\beta]}\left(t^{2} ; x\right)=x^{r}, r=0,1,2 \text {, as } \beta \rightarrow 0
$$

and hence by Korovkin's theorem the proof of theorem (2.1) is complete.

\section{Order of approximation}

TheOREM (3.1). If $f \in C[0, \lambda]$ and $1>\beta^{\prime} / n \geqq \beta \geqq 0$ then

$$
\left|f(x)-P_{n}^{[\beta]}(f ; x)\right| \leqq\left[1+\lambda^{\frac{1}{2}}\left(1+\lambda \beta \beta^{\prime}\right)^{\frac{1}{2}}\right] \cdot \omega\left(1 / n^{\frac{1}{2}}\right),
$$

where $w(\delta)=\sup \left|f\left(x^{\prime \prime}\right)-f\left(x^{\prime}\right)\right| ; x^{\prime}, x^{\prime \prime} \in[0, \lambda], \delta$ being a positive number such that $\left|x^{\prime \prime}-x^{\prime}\right|<\delta$.

Proof. By using the properties of modulus of continuity

$$
\left|f\left(x^{\prime \prime}\right)-f\left(x^{\prime}\right)\right| \leqq w\left(\left|x^{\prime \prime}-x^{\prime}\right|\right) ;
$$




$$
w(\gamma \delta) \leqq(\gamma+1) w(\delta), \gamma>0
$$

and noting the fact that

$$
\sum_{k=0}^{\infty} \omega_{\beta}(k, n x)=1 \text { and } \omega_{\beta}(k, n x) \geqq 0, \forall n, k
$$

it can easily be seen, by the application of Cauchy's inequality, that

$$
\begin{aligned}
\left|f(x)-P_{n}^{[\beta]}(f ; x)\right| & \leqq\left\{1+\frac{1}{\delta} \sum_{k=0}^{\infty} \frac{n x(n x+\beta k)^{k-1}}{k !} e^{-(n x+\beta k)}\left|x-\frac{k}{n}\right|\right\} \omega(\delta) \\
\leqq & \left\{1+\frac{1}{\delta}\left[\sum_{k=0}^{\infty} \frac{n x(n x+\beta k)^{k-1}}{k !} e^{-(n x+\beta k)}\left(x-\frac{k}{n}\right)^{2}\right]^{\frac{1}{2}}\right\} \omega(\delta) .
\end{aligned}
$$

Now by linearity of the operator and by using (2.12), (2.13) and (2.14) we have

$$
\begin{aligned}
& \sum_{k=0}^{\infty} \frac{n x(n x+k \beta)^{k-1}}{k !} e^{-(n x+k \beta)}\left(x-\frac{k}{n}\right)^{2}=x^{2} P_{n}^{[\beta]}(1 ; x)-2 x P_{n}^{[\beta]}(t ; x)+P_{n}^{[\beta]}\left(t^{2} ; x\right) \\
& =x^{2} \beta^{2} /(1-\beta)^{2}+x / n(1-\beta)^{3} \leqq \lambda\left[\lambda \beta \beta^{\prime} /(1-\beta)^{2}+1 /(1-\beta)^{3}\right] / n \\
& \leqq \lambda\left[1+\lambda \beta \beta^{\prime}\right] / n \text {. }
\end{aligned}
$$

Hence using (3.4) in (3.3) and choosing $\delta=1 / \sqrt{ } n$ we prove

$$
\left|f(x)-P_{n}^{[\beta]}(f ; x)\right| \leqq\left[1+\lambda^{\frac{1}{2}}\left(1+\lambda \beta \beta^{\prime}\right)^{\frac{1}{2}}\right] \omega(1 / \sqrt{ } n) .
$$

For $\beta=0$, the expression (3.5) reduces to an inequality for the Szasz-Mirakyan operator obtained earlier by Müller.

Theorem (3.2). If $f \in C^{\prime}[0, \lambda], 1>\beta^{\prime} / n \geqq \beta \geqq 0$, then the following inequality holds

$$
\left|f(x)-P_{n}^{[\beta]}(f ; x)\right| \leqq \lambda^{\frac{1}{2}}\left(1+\lambda \beta \beta^{\prime}\right)^{\frac{1}{2}}\left[1+\lambda^{\frac{1}{2}}\left(1+\lambda \beta \beta^{\prime}\right)^{\frac{1}{2}}\right] \omega_{1}(1 / \sqrt{ } n) / \sqrt{ } n,
$$

where $w_{1}(\delta)$ is the modulus of continuity of $f^{\prime}$.

Proof. For definiteness, we prove the theorem for $f^{\prime}(x) \geqq 0$ but it also applies to $f^{\prime}(x)<0$. By the mean value theorem of differential calculus, it is known that

$$
f(x)-f\left(\frac{k}{n}\right)=\left(x-\frac{k}{n}\right) f^{\prime}(\xi)
$$

where $\xi \equiv \xi_{n, k}(x)$ is an interior point of the interval determined by $x$ and $k / n$. Now

$$
f(x)-f\left(\frac{k}{n}\right) \leqq\left(x-\frac{k}{n}\right)\left[f^{\prime}(\xi)-f^{\prime}(x)\right]+\left[\frac{x}{1-\beta}-\frac{k}{n}\right] f^{\prime}(x) .
$$


Multiplying both sides of the inequality by $x n(x n+\beta k)^{k-1} e^{-(n x+\beta k)} / k$ !, summing over $k$ and using (2.13) we get

$$
\left|f(x)-P_{n}^{[\beta]}(f: x)\right| \leqq \sum_{k=0}^{\infty}\left|x-\frac{k}{n}\right| \frac{n x(n x+\beta k)^{k-1} e^{-(n x+\beta k)}}{k !}\left|f^{\prime}(\xi)-f^{\prime}(x)\right| .
$$

But by (3.1) and (3.2)

$$
\begin{aligned}
\left|f^{\prime}(\xi)-f^{\prime}(x)\right| & \leqq \omega_{1}(|\xi-x|) \leqq\left(1+\frac{1}{\delta}|\xi-x|\right) \omega_{1}(\delta) \\
& \leqq\left(1+\frac{1}{\delta}\left|\frac{k}{n}-x\right|\right) \omega_{1}(\delta)
\end{aligned}
$$

where $\delta$ is a positive number not depending on $k$.

A use of this in (3.6) gives

$$
\begin{aligned}
\left|f(x)-P_{n}^{[\beta]}(f ; x)\right| \leqq & \left\{\sum_{k=0}^{\infty}\left|x-\frac{k}{n}\right| \frac{n x(n x+\beta k)^{k-1}}{k !} e^{-(n x+\beta k)}\right. \\
& \left.+\frac{1}{\delta} \sum_{k=0}^{\infty}\left(x-\frac{k}{n}\right)^{2} \frac{n x(n x+\beta k)^{k-1}}{k !} e^{-(n x+\beta k)}\right\} \omega_{1}(\delta) .
\end{aligned}
$$

Hence by applications of Cauchy's inequality and (3.4)

$$
\left|f(x)-P_{n}^{[\beta]}(f ; x)\right| \leqq \frac{\lambda^{\frac{1}{2}}\left(1+\lambda \beta \beta^{\prime}\right)^{\frac{1}{2}}}{n^{\frac{1}{2}}}\left[1+\frac{\lambda^{\frac{1}{2}}\left(1+\lambda \beta \beta^{\prime}\right)^{\frac{1}{2}}}{\delta n^{\frac{1}{2}}}\right] \omega_{1}(\delta) .
$$

Choosing $\delta=1 / \sqrt{ } n$, theorem (3.2) is proved.

We may put $\beta=0, \delta=1 / \sqrt{ } n$ in (3.7) to get the expression for Szasz-Mirakyan operator. The substitutions reduce $(3.7)$ to

$$
\left|f(x)-P_{n}^{[\beta]}(f ; x)\right| \leqq \frac{1}{\sqrt{ } n}(\lambda+\sqrt{ } \lambda) \omega_{1}(1 / \sqrt{ } n) ; x \in\left[\begin{array}{ll}
0 & \lambda
\end{array}\right]
$$

in agreement with Stancu [12].

\section{References}

[1] E. W. Cheney and A. Sharma, 'On a Generalization of Bernstein Polynomials', Rev. Mat. Univ. Parma (2) 5 (1964), 77-84.

[2] P. C. Consul and G. C. Jain, 'On a Generalized Poisson Distribution', Submitted (1970).

[3] G. C. Jain and S. P. Pethe, 'On a Generalization of Szasz-Mirakyan operator', Mathematica (Cluj) (2) 35 (1970), 313-318.

[4] A. Jakimovski and D. Leviatan, 'Generalized Bernstein Polynomials', Math. Z. 93 (1966), $411-426$.

[5] J. L. W. V. Jensen, 'Sur une identité, d'Abel et sur d'autres formules analogues', Acta Math. 26, (1902), 307-318.

[6] P. P. Korovkin, Linear Operators and Approximation Theory (translated from Russian edition of 1959, Delhi, 1960.) 
[7] A. Meir and A. Sharma, 'Approximation Methods by Polynomials and Power Series', Koninkl. Nederl. Akademie Van Wetenschappen. Amsterdam, Reprinted from Proceedings, Series A, 70, No. 4 and Indag. Math. 29 (1967), 77-84.

[8] W. Meyer-König and K. Zeller, 'Bernsteinsche Potenzreihen', Studia Math. 19 (1960), 89-94.

[9] G. Mirakyan, 'Approximation des Fonctions Continues au Moyen de Polynomes de la Forme', Dokl. Akad. Nauk. SSSR 31 (1941), 201-205.

[10] M. Müller, Die Folge der Gamma Operatoren, Dissertation, Stuttgart, 1967).

[11] S. P. Pethe and G. C. Jain, 'Approximation of functions by Bernstein type operator', Canad. Math. Bull., to appear.

[12] D. D. Stancu, 'Approximation of functions by a New Class of Linear Polynomial Operators', Revue Roumaine de Mathématique Pures et Appliquées 8 (XIII) (1968), 1173-1994.

[13] O. Szasz, 'Generalization of S. Bernstein's Polynomials to the Infinite Interval', $J$. of Research of the Nat. Bur. of Standards, 45, (1950) 239-245; Collected Mathematical Works (Cincinnati 1955, 1401-1407).

University of Calgary

Alberta, Canada 